PROCEEDINGS OF THE

AMERICAN MATHEMATICAL SOCIETY

Volume 125, Number 3, March 1997, Pages 663-668

S 0002-9939(97)03637-X

\title{
MORDELL-WEIL GROUPS OF THE JACOBIAN OF THE 5-TH FERMAT CURVE
}

\author{
PAVLOS TZERMIAS
}

(Communicated by William W. Adams)

\begin{abstract}
Let $J_{5}$ denote the Jacobian of the Fermat curve of exponent 5 and let $K=Q\left(\zeta_{5}\right)$. We compute the groups $J_{5}(K), J_{5}\left(K^{+}\right), J_{5}(Q)$, where $K^{+}$is the unique quadratic subfield of $K$. As an application, we present a new proof that there are no $K$-rational points on the 5 -th Fermat curve, except the so called "points at infinity".
\end{abstract}

\section{INTRODUCTION}

Let $F_{5}$ denote the complete non-singular curve over $Q$ with projective equation

$$
X^{5}+Y^{5}+Z^{5}=0 .
$$

We denote by $J_{5}$ the Jacobian of $F_{5}$. Let $\zeta_{5}$ be a primitive 5 -th root of unity in $\bar{Q}$ and let $K=Q\left(\zeta_{5}\right)$. Also let $K^{+}$denote the unique quadratic subfield of $K$. As Faddeev $([2])$ has shown, the group $J_{5}(K)$ of $K$-rational points of $J_{5}$ is finite. In this paper, we will determine the structure of this group, as well as of its subgroups $J_{5}\left(K^{+}\right)$and $J_{5}(Q)$.

There are 15 points $(X, Y, Z)$ on $F_{5}$ for which one of $X, Y, Z$ is 0 , namely the points $a_{j}=\left(0, \epsilon \zeta_{5}^{j}, 1\right), b_{j}=\left(\epsilon \zeta_{5}^{j}, 0,1\right), c_{j}=\left(\epsilon \zeta_{5}^{j}, 1,0\right)$, where $\epsilon=e^{\pi i / 5}, \zeta_{5}=\epsilon^{2}$ and $0 \leq j \leq 4$. These points will be called the points at infinity on $F_{5}$.

Let $J_{5}^{\infty}$ be the subgroup of $J_{5}$ consisting of those divisor classes of degree 0 which contain a divisor supported on the points at infinity. Rohrlich ([7]) has completely determined the structure of the group $J_{5}^{\infty}$. It is isomorphic to $(Z / 5 Z)^{8}$.

The main result of this paper is:

Proposition. In the above notation, we have $J_{5}(K)=J_{5}^{\infty}$.

As a result of this proposition, we will also deduce:

Corollary 1. $J_{5}\left(K^{+}\right)$is isomorphic to $(Z / 5 Z)^{5}$.

Corollary 2. $J_{5}(Q)$ is isomorphic to $(Z / 5 Z)^{2}$.

Corollary 3. The only $K$-rational points on $F_{5}$ are the points at infinity.

Remark. It should be noted that Fermat's Last Theorem for the exponent 5 is implied by Corollary 3.

Received by the editors November 5, 1994 and, in revised form, September 1, 1995.

1991 Mathematics Subject Classification. Primary 14H25, 14G05; Secondary 11D41.

(C)1997 American Mathematical Society 


\section{THE 5-PRIMARY PART OF $J_{5}(K)$}

In this Section, we will determine the 5-primary part of $J_{5}(K)$. Let $\sigma, \tau$ be the automorphisms of $F_{5}$ given by

$$
\begin{aligned}
& \sigma(X, Y, Z)=\left(\zeta_{5} X, Y, Z\right), \\
& \tau(X, Y, Z)=\left(X, \zeta_{5} Y, Z\right) .
\end{aligned}
$$

Let $a, b$ be positive integers such that $1 \leq a, b, a+b \leq 4$. Consider the quotient curve $F_{a, b}=F_{5} /\left\langle\sigma^{b} \tau^{-a}\right\rangle$ and let $J_{a, b}$ be the Jacobian of $F_{a, b}$.

The curve $F_{a, b}$ is defined over $Q$ and has the affine singular equation

$$
y^{5}=x^{a}(1-x)^{b} .
$$

It has a birational automorphism given by $(x, y) \mapsto\left(x, \zeta_{5} y\right)$, which induces an endomorphism of $J_{a, b}$, which will also be called $\zeta_{5}$. Now consider the element $\pi=\zeta_{5}-1$ of $\operatorname{End}\left(J_{a, b}\right)$.

In this Section, we will concentrate on $J_{1,3}$ only and for simplicity of notation, we will write $J=J_{1,3}$. Let $Q$ be a $\pi^{2}$-division point on $J$ which is not a $\pi$-division point. We also assume that the complex conjugate of $Q$ equals $-Q$. Then $\operatorname{Lim}([6])$ has shown that $J_{5}$ is isomorphic to $J^{2} \times B$ over $K$, where $B$ is the abelian variety which is the quotient of $J$ by the subgroup generated by $(1+3 \pi) Q$. Let $\phi: J \longrightarrow B$ be the corresponding isogeny (defined over $K$ ), with kernel equal to $\langle(1+3 \pi) Q\rangle$.

Now, 5 does not divide the class number of $K^{+}$; therefore, by Greenberg's result (see [3]), the 5-primary part of $J(K)$, denoted by $(J(K))_{5-\text { power }}$, equals the kernel of the isogeny $\pi^{3}$ of $J$. We write the kernel of $\pi^{3}$ as $J\left[\pi^{3}\right]$.

So we only need to determine $(B(K))_{5-\text { power }}$.

We will prove the following two lemmas:

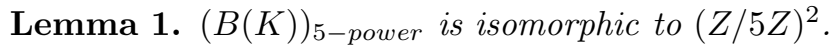

Lemma 2. $\left(J_{5}(K)\right)_{5-\text { power }}=J_{5}^{\infty}$.

We will prove both lemmas together. We proceed as follows:

Let $D_{0}$ be a point in $B(K)$ of exact order $5^{r}$, for some non-negative integer $r$. Let $\sigma$ be in $\operatorname{Gal}(\bar{K} / K)$. Lift to a point $D$ in $J$. Since $D_{0}$ is defined over $K$, we have $D^{\sigma}-D=a(1+3 \pi) Q$, for some integer $a$.

Then $\pi^{2} D^{\sigma}-\pi^{2} D=0$, because $Q$ is a $\pi^{2}$-division point. Now $\pi^{\sigma}=\pi$; therefore $\left(\pi^{2} D\right)^{\sigma}=\pi^{2} D$. This is true for all $\sigma$ in $\operatorname{Gal}(\bar{K} / K)$; therefore $\pi^{2} D$ is in $J(K)$. Also, $5^{r} D_{0}=0$, so $5^{r} D$ is in $\langle(1+3 \pi) Q\rangle$; hence $5^{r} \pi^{2} D=0$. Therefore, $\pi^{2} D$ is

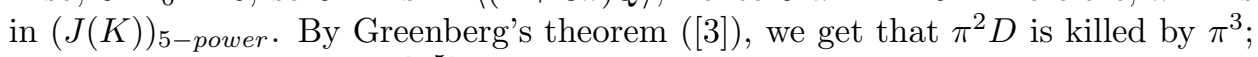
hence $r \leq 1$ and $D$ is in $J\left[\pi^{5}\right]$. We have thus proved:

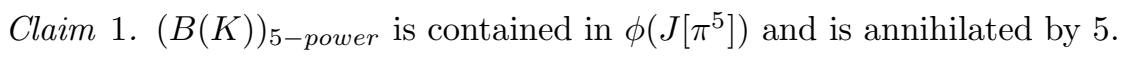

Now suppose that $D$ is in $J\left[\pi^{5}\right]-J\left[\pi^{4}\right]$ such that $\phi(D)$ is in $(B(K))_{5-\text { power }}$. By claim 1 , we get $5 \phi(D)=0$, so $5 D=a(1+3 \pi) Q$, for some integer $a$. Then $5 \pi D=a P$, where $P=\pi Q$.

Observe that $J\left[\pi^{4}\right]=J[5]$; therefore $5 \pi D=0$, hence $a P=0$. Since $Q$ is not a $\pi$-division point, we get that 5 divides $a$, hence $5 D=0$. This means that $D$ is in $J\left[\pi^{4}\right]$, a contradiction. Therefore, we have proved:

Claim 2. $(B(K))_{5-\text { power }}$ is a subgroup of $\phi\left(J\left[\pi^{4}\right]\right)$. 
Now suppose that $D$ is in $J\left[\pi^{4}\right]-J\left[\pi^{3}\right]$ such that $\phi(D)$ is in $(B(K))_{5-\text { power }}$. Let $\sigma$ be in $\operatorname{Gal}(\bar{K} / K)$. Then $D^{\sigma}-D=a(1+3 \pi) Q$, for some integer $a$. Therefore, $\pi D^{\sigma}-\pi D=a P$, where, again, $P=\pi Q$. But $\pi D$ is in $J\left[\pi^{3}\right]$; therefore, by [4], we have $\pi D^{\sigma}=(\pi D)^{\sigma}=\pi D$. Hence $a P=0$, so, since $Q$ is not a $\pi$-division point, we get that 5 divides $a$, so $D^{\sigma}=D$. This being true for all $\sigma$ in $\operatorname{Gal}(\bar{K} / K)$, we conclude that:

Claim 3. $(B(K))_{5-\text { power }}$ is a subgroup of $\phi\left(J\left[\pi^{3}\right]\right)$.

Claim 3, together with a cardinality argument, shows that the order of $(B(K))_{5-\text { power }}$ divides $5^{2}$.

Therefore, since $J_{5}$ and $J^{2} \times B$ are isomorphic over $K$, we get that $\left(J(K)_{5-\text { power }}\right.$ has order dividing $5^{8}$. On the other hand, Rohrlich ([7]) has proved that $J_{5}^{\infty}$ is isomorphic to $(Z / 5 Z)^{8}$. Also, evidently, $J_{5}^{\infty}$ is contained in $(J(K))_{5-\text { power }}$.

This proves Lemmas 1 and 2 .

\section{NON-EXISTENCE OF $l$-TORSION FOR $l \neq 5$}

Consider the abelian variety $J_{a, b}$ as in Section 2 . Let $f_{5}(X)$ be the 5 -th cyclotomic polynomial and let $J_{a, b}^{n e w}$ denote the quotient of $J_{a, b}$ by the abelian subvariety $f_{5}(g) J_{a, b}$, where $g=\sigma^{b} \tau^{-a}$. Then $J_{a, b}^{n e w}$ is defined over $Q$.

Claim 4. We have $J_{a, b}^{\text {new }}=J_{a, b}$.

Indeed, we have $\operatorname{Ker}(g-1)=0$ and $g^{5}=1$ on $J_{a, b}$; therefore we get that $f_{5}(g) J_{a, b}=0$, which proves the claim.

Now, using results of Coleman ([1]), we can show:

Claim 5. There is no $l$-torsion in $J_{a, b}(K)$, for $l \neq 5$.

Indeed, let $l$ be a prime, $l \neq 5$. Suppose $T$ is an $l$-torsion point on $J_{a, b}=J_{a, b}^{n e w}$. By Proposition 10 in [1], the field extension $Q(T) / Q$ is ramified above $l$ unless $l=2$ and $J_{a, b}^{n e w}$ is ordinary at 2 . However, by Corollary 13.1 in $[1], J_{a, b}^{n e w}$ is not ordinary at 2 in the case of the Fermat quintic.

Therefore, we deduce that the field extension $Q(T) / Q$ is ramified above $l$, whenever $l \neq 5$ and $T$ is an $l$-torsion point on $J_{a, b}$. Since $K / Q$ is unramified above $l$, the point $T$ can never be $K$-rational, so the claim follows.

Now we can show:

Lemma 3. Let $l$ be a prime, $l \neq 5$. Then $J_{5}(K)$ has no $l$-torsion.

Proof. We have an isogeny defined over $Q$

$$
f: J_{5} \longrightarrow J_{1,1} \times J_{1,2} \times J_{1,3} .
$$

Also let

$$
\hat{f}: J_{1,1} \times J_{1,2} \times J_{1,3} \longrightarrow J_{5}
$$

be the dual isogeny. Then $\hat{f} \circ f$ equals multiplication by 5 on $J_{5}$ (see for example $[5])$.

If $T$ is an $l$-torsion point in $J_{5}(K)$, then $f(T)$ is an $l$-torsion point in $J_{1,1}(K) \times$ $J_{1,2}(K) \times J_{1,3}(K)$.

By Claim 5, we get $f(T)=0$; therefore $T$ is in $\operatorname{Ker}(f)$, which is contained in $J_{5}[5]$. Hence, $T$ is also a 5 -torsion point; therefore $T=0$. This proves Lemma 3 . 
The proposition of the introduction now follows from Lemmas 2 and 3.

Finally, we will compute $\operatorname{Ker}(f)$. Consider the group $G$ of divisor classes of degree 0 on $F_{5}$ which are represented by a linear combination of the divisors $a_{j}-a_{2}, b_{j}-b_{2}$, $c_{j}-c_{2}$, for $j \neq 2,0 \leq j \leq 4$. Then:

Lemma 4. $\operatorname{Ker}(f)=G$.

Proof. It is evident that $G$ is contained in $\operatorname{Ker}(f)$ and it follows from the work of Rohrlich ([7]) that $G$ has cardinality $5^{6}$.

Since $\hat{f} \circ f=5$ and the cardinalities of $\operatorname{ker}(\hat{f})$ and $\operatorname{ker}(f)$ are the same, the lemma follows.

\section{The Structure of $J_{5}\left(K^{+}\right)$And $J_{5}(Q)$}

Now we will prove Corollaries 1 and 2 of the introduction. Recall that $J_{5}^{\infty}$ denotes the divisor classes of degree 0 on $J_{5}$ that can be represented by a divisor supported on the points at infinity.

Rohrlich ([7]) has shown that every element of $J_{5}^{\infty}$ is represented by a linear combination of the divisors $a_{j}-a_{2}, b_{j}-b_{2}, c_{j}-c_{2}, a_{2}-c_{2}, b_{2}-c_{2}$, for $j \neq 2$, $0 \leq j \leq 4$

So take any divisor $\bar{D}$ in $J_{5}(K)$. By the proposition of the introduction, we can choose a representative $D$ of the form

$$
D=s\left(a_{2}-c_{2}\right)+t\left(b_{2}-c_{2}\right)+\sum_{j=0}^{4}\left(x_{j}\left(a_{j}-a_{2}\right)+y_{j}\left(b_{j}-b_{2}\right)+z_{j}\left(c_{j}-c_{2}\right)\right),
$$

for integers $x_{j}, y_{j}, z_{j}, s, t$.

Now $K=Q\left(\zeta_{5}\right)=Q(\epsilon)$. Let $\alpha$ be the generator of $\operatorname{Gal}(K / Q)$ given by $\alpha(\epsilon)=\epsilon^{3}$. If $\bar{D}$ is in $J_{5}(Q)$, then we must have that $D^{\alpha}-D$ is principal. But

$$
D^{\alpha}-D=\sum_{j=0}^{4}\left(x_{j}\left(a_{3 j+1}-a_{j}\right)+y_{j}\left(b_{3 j+1}-b_{j}\right)+z_{j}\left(c_{3 j+1}-c_{j}\right)\right) .
$$

Now, by Corollary 1 to Theorem 2 of [7] and a tedious calculation (not presented here), we get that $D^{\alpha}-D$ is principal if and only if $D-s\left(a_{2}-c_{2}\right)-t\left(b_{2}-c_{2}\right)$ is a principal divisor.

This proves Corollary 1.

The proof of Corollary 2 is similar. We only need to replace the automorphism $\alpha$ by the automorphism $\beta$ given by $\beta(\epsilon)=\epsilon^{-1}$.

\section{The SET $F_{5}(K)$}

As an application of the proposition, we will now prove Corollary 3.

Consider again the curve $F_{1,3}$ and the abelian variety $J=J_{1,3}$, as in Section 2 . We have the quotient map

$$
g: F_{5} \longrightarrow F_{1,3},
$$

which induces a map $g_{*}$ on the divisors of degree 0 and hence a map (also denoted by $g_{*}$ )

$$
g_{*}: J_{5} \longrightarrow J_{1,3} \text {. }
$$

Similarly, we have the induced pullback map $g^{*}$ on the divisors of degree 0 on $F_{1,3}$ and hence a map

$$
g^{*}: J_{1,3} \longrightarrow J_{5}
$$


It is easy to see that if $D$ is a divisor of degree 0 on $F_{5}$ then we have

$$
g^{*}\left(g_{*}(D)\right)=\sum_{j=0}^{4}\left(\sigma^{2} \tau\right)^{j} D .
$$

We have the points $P_{0}=(0,0,1), P_{1}=(1,0,1)$ and $P_{\infty}=(1,0,0)$ on $F_{1,3}$. Observe that $g\left(a_{j}\right)=P_{0}, g\left(b_{j}\right)=P_{1}, g\left(c_{j}\right)=P_{\infty}$, for $0 \leq j \leq 4$.

Now if $P$ is a $K$-rational point on $F_{5}$, which is not a point at infinity, let $D$ be the equivalence class of the divisor $P-c_{2}$.

$D$ is in $J_{5}(K)$, so, by our proposition, we get that $g_{*}(D)$ is represented by a divisor of degree 0 supported on the points $P_{0}, P_{1}, P_{\infty}$. By [4], any such divisor is linearly equivalent to a multiple of $P_{0}-P_{\infty}$. Since $g^{*}\left(P_{0}-P_{\infty}\right)=\operatorname{div}(X / Z)$, we conclude that $g^{*}\left(g_{*}(D)\right)$ is linearly equivalent to 0 . But

$$
g^{*}\left(g_{*}\left(P-c_{2}\right)\right)=\sum_{j=0}^{4}\left(\sigma^{2} \tau\right)^{j} P-\sum_{j=0}^{4} c_{j},
$$

which cannot be principal, by Lemma 2.1 of [4] (where we take $d=1$ and $u=2$ ).

\section{Final REMARKS}

1. Greenberg has shown ([3]) that in the case of the quotient curves $F_{a, b}$, there exists a positive integer $s$ such that the group $J_{a, b}(K)$ equals the kernel of the isogeny $\pi^{s}$ of $J_{a, b}$. As a result of the proposition proved in this paper, we see that this cannot be the case for the Fermat curve $F_{5}$. Indeed, the kernel of the isogeny $\pi$ of $J_{5}$ has cardinality $5^{3}$, so the kernel of $\pi^{s}$ has cardinality $5^{3 s}$, which can never equal $5^{8}$.

2. Let $\zeta_{6}$ be a primitive 6 -th root of unity in $\bar{Q}$ and let $P=\left(\zeta_{6}, \zeta_{6}^{-1}, 1\right)$ and $\bar{P}=\left(\zeta_{6}^{-1}, \zeta_{6}, 1\right)$ on $F_{5}$. Gross and Rohrlich ([4]) have considered the divisor $D=$ $P+\bar{P}-a_{2}-b_{2}$ on $F_{5}$. It is evident that $D$ (not just the divisor class of $D$ ) is defined over $Q$. Then Corollary 2 implies that the divisor class of $D$ is a linear combination of the divisor classes of $a_{2}-c_{2}$ and $b_{2}-c_{2}$. Indeed, a little search shows that

$$
D+2\left(a_{2}-c_{2}\right)+2\left(b_{2}-c_{2}\right)=\operatorname{div}(x+y-1)-\operatorname{div}(x+y),
$$

where $x=X / Z, y=Y / Z$.

\section{ACKNOWLEDGMENTS}

This paper will constitute part of my doctoral dissertation at Berkeley. I am indebted to Robert F. Coleman for his support and encouragement during the course of this work.

\section{REFERENCES}

[1] R. F. Coleman, Torsion points on Abelian etale coverings of $P^{1}-\{0,1, \infty\}$, Transactions of the AMS, 311, No. 1 (1989), 185-208. MR 90a:11064

[2] D. K. Faddeev, On the divisor class groups of some algebraic curves, Soviet Math. Dokl. 2 (1961), 67-69. MR 24:A723

[3] R. Greenberg, On the Jacobian variety of some algebraic curves, Compositio Math. 42 (1981), 345-359. MR 82j: 14036

[4] B. Gross and D. Rohrlich, Some results on the Mordell-Weil group of the Jacobian of the Fermat curve, Invent. Math. 44 (1978), 201-224. MR 58:10911 
[5] S. Lang, Introduction to algebraic and abelian functions, GTM 89, Springer-Verlag, New York-Berlin-Heidelberg. MR 48:6122

[6] C. H. Lim, The geometry of the Jacobian of the Fermat curve of exponent five, Journal of Number Theory 41 (1991), 102-115. MR 93j:14030

[7] D. Rohrlich, Points at infinity on the Fermat curves, Invent. Math. 39 (1977), 95-127. MR 56:367

Department of Mathematics, University of California, Berkeley, California 94720

E-mail address: tzermias@math. berkeley . edu

Current address: Centre de Recerca Matemàtica, Institut d'Estudis Catalans, Apartat 50, E08193 Bellaterra, Spain

Current e-mail address: tzermias@crm.es 